\title{
BM] open Suicide-related events in young people following prescription of SSRIs and other antidepressants: a self-controlled case series analysis
}

\author{
Linda P M M Wijlaars, ${ }^{1}$ Irwin Nazareth, ${ }^{1}$ Heather J Whitaker, ${ }^{2}$ Stephen J W Evans, ${ }^{3}$ \\ Irene Petersen ${ }^{1}$
}

To cite: Wijlaars LPMM, Nazareth I, Whitaker HJ, et al. Suicide-related events in young people following prescription of SSRIs and other antidepressants: a selfcontrolled case series analysis. BMJ Open 2013;3: e003247. doi:10.1136/ bmjopen-2013-003247

- Prepublication history and additional material for this paper is available online. To view these files please visit the journal online (http://dx.doi.org/10.1136/ bmjopen-2013-003247).

Received 17 May 2013 Revised 5 August 2013 Accepted 9 August 2013

${ }^{1}$ Department of Primary Care and Population Health, University College London Medical School, London, UK ${ }^{2}$ Department of Mathematics and Statistics, The Faculty of Mathematics, Computing and Technology, The Open University, Milton Keynes, Buckinghamshire, UK ${ }^{3}$ Department of Medical Statistics, London School of Hygiene and Tropical Medicine, London, UK

Correspondence to Ms Linda P M M Wijlaars; linda.wijlaars.10@ucl.ac.uk

\section{ABSTRACT}

Objectives: We aimed to examine the temporal association between selective serotonin reuptake inhibitors (SSRI) and tricyclic antidepressant (TCA) prescriptions and suicide-related events in children and adolescents.

Design: Self-controlled case series.

Setting: Electronic health records were used from 479 general practices in The Health Improvement Network (THIN) UK primary care database from 1995 to 2009.

Participants: 81 young people aged $10-18$ years with a record of completed suicide, 1496 who attempted suicide, 1178 with suicidal ideation and 2361 with intentional self-harm.

Main outcome measures: Incidence Rate Ratios (IRRs) for completed and attempted suicide, suicidal ideation and intentional self-harm.

Results: For non-fatal suicide-related behaviour, IRRs were similar for the time the person was prescribed either SSRIs or TCAs: IRRs increased during preexposure, peaked on prescription day, were stable up to the fourth prescription-week, and decreased after the prescriptions were stopped. For both types of antidepressants, IRRs were lower or similar to preexposure levels during the period of prescription. For SSRIs, there was an increase in the IRR for completed suicide on the day of prescription ( $N=5$; IRR=42.5, $95 \% \mathrm{Cl} 4.5$ to 403.4 ), and during the fourth week of SSRI prescription ( $\mathrm{N}=2$; IRR $=11.3,95 \% \mathrm{Cl} 1.1$ to 115.6).

Conclusions: We found that a very small number of young people were prescribed antidepressants and that there was an absence of a sustained increase in rates of suicide-related events in this group. There were no systematic differences between the association of TCAs and SSRIs and the incidence risk ratios for attempted suicide, suicidal ideation or intentional self-harm and, apart from the day of prescription, rates did not exceed pre-exposure levels. The pattern of IRR for suicide for SSRIs was similar to that found in non-fatal suiciderelated events. Our results warrant a re-evaluation of the current prescription of SSRIs in young people. We recommend the creation of a pragmatic registry for active pharmacovigilance.

\section{ARTICLE SUMMARY}

Strengths and limitations of this study

- Only a limited number of young people had a prescription for an antidepressant in the year before their suicide-related event, making it difficult to interpret the findings of this study.

- The self-controlled case series method inherently controls for time-independent variables such as genetics, location and socio-economic status.

- Changes in depression severity are poorly recorded over time, which is a limitation.

\section{INTRODUCTION}

Between $1 \%$ and $6 \%$ of adolescents in the community suffer from major depressive disorder (MDD). ${ }^{1}$ In addition, suicide is the third leading cause of death in 15-year-olds to -19 -year-olds at $6.9 / 100000$ population, and the fourth in 10-year-olds to 14-year-olds at $0.9 / 100000$ population. ${ }^{2}$ This calls for safe and effective depression treatments in this age group. As tricyclic antidepressants (TCAs) lack efficacy for depression treatment in this age group and have a poor side-effect profile, ${ }^{3}$ selective serotonin reuptake inhibitors (SSRIs) are the most commonly prescribed pharmacological treatment for children and adolescents. ${ }^{4}$

However, there has been concern that SSRIs might be associated with an increased risk of suicide-related events in paediatric patients. Results from clinical trials led the Expert Working Group of the Committee on Safety of Medicines (CSM) to advise against initiation of treatment with selective serotonin inhibitors (SSRIs) for childhood depression in the UK in December 2003. ${ }^{5}$ Fluoxetine, the only drug which is licensed to treat depression in young people in the UK, was exempted from this advice following a review that concluded that there was a 
favourable balance of benefits and risk. ${ }^{6}$ The US Food and Drug Administration (FDA) issued similar advice in $2004 .^{7}$

There is inconsistent evidence of an increased rate of suicide-related events and intentional self-harm associated with SSRIs. ${ }^{8}$ Data from randomised controlled trials in adolescents and young adults report an increased risk of suicide-related events. ${ }^{9}$ Part of this difference appears to depend on the methodology used. If suicide-related events were ascertained using the method of 'adverse events', there was a small but significant increase in suicidal ideation. However, if the studies used rating scales to assess suicide-related events, most studies showed an improvement in suicide-related events.

The results from these trials should be interpreted with caution, as they were not primarily designed to measure suicide-related events and it would be unethical to do so using placebo as a control. ${ }^{10} 11$ Moreover, none of these trials on SSRIs recruited from a general population setting and completed suicides have occurred in any studies. 9

Observational studies in young people have found mixed results: some indicate that SSRIs protect from suicide-related events ${ }^{12}$; others find no effect ${ }^{13} 14$ or an increase in risk of suicide-related events. ${ }^{15} 16$ These studies, however, have methodological limitations including small numbers, high attrition rates and, most importantly, confounding by severity.

We have previously shown that rates for SSRI prescriptions in children and adolescents increased between 2005 and 2009. ${ }^{4}$ Neither TCAs nor SSRIs are considered appropriate first-line treatment by the National Institute for Clinical Excellence (NICE) for depression in children and adolescents. Given the risk of death in overdose, the lack of efficacy in children and the side effects associated with them, a prescriber would be less likely to prescribe TCAs in preference to SSRIs for a person at risk of suicide-related events. ${ }^{17}$

It is only when children and adolescents do not respond to psychological treatment that treatment with SSRIs should be considered. ${ }^{6}$ It is therefore important to reassess the risks of existing clinical data to inform future practice. We aimed to assess the temporal association between the risk of completed suicide, attempted suicide, suicidal thoughts, intentional self-harm and antidepressant prescription in adolescents, comparing SSRIs and TCAs and correcting for age and gender, using a large UK primary care database.

\section{METHODS}

\section{Data source}

We used data from The Health Improvement Network (THIN) primary care database, including information from UK primary care data prospectively recorded between 1 January 1995 and 31 December 2009. THIN includes anonymised general practice records on more than 9 million patients from 479 practices in the UK and is one of the largest primary care databases available internationally. It is broadly representative of the UK general practice population in terms of demographics and consultation behaviour. ${ }^{18}$ Data on diagnoses, interventions, symptoms and referrals to secondary care are electronically recorded as Read codes, a hierarchical coding system used in UK primary care.$^{19}$ All prescriptions are also electronically recorded. Clinical diagnoses recorded using Read codes have recently been shown to be accurate compared with other reliable sources. ${ }^{20}$

\section{Study population}

This study included a cohort of young people and adolescents, aged 10-18 years, who had a record of a suicide-related event. Patients were included if they were registered with a practice for at least 6 months between January 1995 and December 2009. Patients were followed up from the latest date they registered at the general practitioner (GP), 1 January 1995, or their 10th birthday, until (1) 31 December 2009, (2) their 19th birthday, (3) the date of death or (4) the date they left the practice.

\section{Measurements}

\section{Outcome}

We identified completed suicides using relevant Read codes that were confirmed by a date of death within 2 weeks of the suicide event date. We searched a cause of death, if available. The list of codes was an updated version of a published suicide code list. ${ }^{21}$ To make sure that we did not miss any suicides, we extracted medical records on all young people who died between the ages of 10 and 18 and examined the free text if there was no clear cause of death (eg, childhood cancer or a traffic accident) for possible suicides. We excluded cases where there was doubt whether the death was due to suicide (ie, 12 deaths which received an open verdict by the coroner). Of these potential suicides, one patient had records of TCA prescriptions, whereas four had records of SSRI prescriptions in the last year. A suicide attempt, suicidal ideation and self-harm were identified using a Read code list that was developed in line with the published methods and reviewed by a GP (IN). ${ }^{22}$

\section{Exposure}

We used British National Formulary (BNF) codes representing antidepressants. ${ }^{23}$ We classified antidepressants as TCAs, SSRIs and other antidepressants according to BNF. We excluded TCAs that were prescribed for nocturnal enuresis or neuropathic pain. Comparing prescription data in THIN to dispensing data from NHS Prescription Services showed that the mean practice redemption rate (the percentage of recorded prescriptions which were dispensed) was as high as $96.7 \%$ for antidepressants. ${ }^{24}$

We then identified the separate episodes of antidepressant prescription for each individual. To 
constitute a new episode of antidepressant prescriptions, there had to be a preceding gap of at least 3 months of no prescriptions. We choose 3 months as a prescription supply of antidepressants is typically 1 month or less, and therefore a gap of at least 3 months between prescriptions would quite likely represent a new episode of antidepressant prescriptions (although not necessarily a new episode of depression). If a person switched from one drug to another within 3 months, this would not constitute a new episode.

\section{Covariates}

We extracted information on gender, age and social deprivation score (Townsend quintiles). Patients who were prescribed TCAs for nocturnal enuresis (as confirmed by Read codes) were excluded. Finally, we considered consultation behaviour as a confounder. Patients who consult infrequently with their GP, for example, only when their prescription has run out, might not have correctly timed records of all their events. To correct for this, we conducted a sensitivity analysis where we only included patients who consulted at least once a week during the month after their first antidepressant prescription.

\section{Statistical analysis}

The self-controlled case series (SCCS) method

We calculated incidence rate ratios (IRRs-calculated by dividing the incidence rate in the exposed period by the incidence rate in the control period) for completed suicide and suicide-related events using the selfcontrolled case series (SCCS) method. ${ }^{25}$ The SCCS method was developed to investigate associations between acute outcomes and transient exposures, using only data on cases. Since each case served as his/her own control, the case-series method inherently accounts for confounding factors that do not change over the observation period (such as variables related to genetics, socioeconomic status and gender). Using a Poisson model, IRRs can be calculated for any number of predefined risk periods associated with the exposure, using the time outside of these risk periods (the time when a subject is unexposed) as a reference. A major advantage of SCCS is that it can have high efficiency relative to the retrospective cohort method from which it is derived. As suicide-related events are rare, even in depressed persons, the SCCS method is particularly suited for assessing its association with antidepressants, as it requires a much smaller number of persons to be included. Moreover, in using a self-controlled design, we circumvent the problem of selecting appropriate controls that cohort or case-control designs encounter. In the case of suicide-related events and antidepressants, this has proven crucial as patients who are diagnosed as depressed but do not receive antidepressants might differ from patients who are receiving antidepressants in severity of depression, a major confounder in the association studied.
The SCCS method is limited in that it only works for non-recurrent events when the event risk is small over the observation period, which is the case for completed suicide. Also, it requires variability in the age at the time of the event: if all events were to happen at exactly the same age (which is not the case in our study), then the method would fail. Finally, the method only produces estimates of relative incidence, rather than absolute incidence.

\section{Suicide attempts, suicidal ideation and self-harm}

For the analyses on suicide attempts, suicidal ideation and self-harm, we used an adapted version of the standard SCCS method, allowing for repeated exposures and events and correcting by 1 year age groups. ${ }^{25} 26$ By including a pre-exposure period, we corrected our estimates for eventdependent exposure. If the probability of exposure to antidepressants is changed from baseline after an event, it follows that the probability of the occurrence of an event is also changed in the immediate pre-exposure period. Including a pre-exposure period removes this time from the baseline and corrects the estimates accordingly.

Participants who went on to commit suicide were excluded from these analyses. Using the SCCS method, the IRR for the three outcomes was estimated during 14 different risk periods (figure 1): baseline (or unexposed to antidepressants); four 1-month pre-exposure periods, the last of which is the reference; the day of prescription; four 1-week early exposure periods; a period of variable length to cover the remainder of the period of exposure to the antidepressant for that episode; and three 1-month periods of washout after the end of the antidepressant episode. We included separate 1-week periods at the start of the prescription as it is known that antidepressants (especially SSRIs) take this amount of time to have an effect. ${ }^{27}$ We also compared the effects of individual antidepressants on the IRR of the three suicide-related event outcomes.

\section{Suicide}

We used an adapted method (SCCS for censored post-event exposures) to assess the effects of antidepressants on childhood suicide as the original approach cannot deal with deaths. ${ }^{28}$ This method takes account of the early cessation of the observation period due to deaths and accordingly corrects the IRR estimates. We corrected for age by creating four age groups: 10-12, 13-14, 15-16 and 17-18-year-olds. We estimated IRRs for the same risk periods as for the other outcomes, without the four pre-exposure risk periods, and used the baseline (time unexposed to antidepressants) as a reference.

All analyses were conducted with the use of Stata software, V.12.1 (Stata Corp, College Station, Texas). The scheme for THIN to obtain and provide anonymous patient data to researchers was approved by the National Health Service South-East Multicenter Research Ethics Committee (MREC) in 2002 and scientific approval for this study was obtained from CMD Medical Research's Scientific Review Committee in May 2011. 


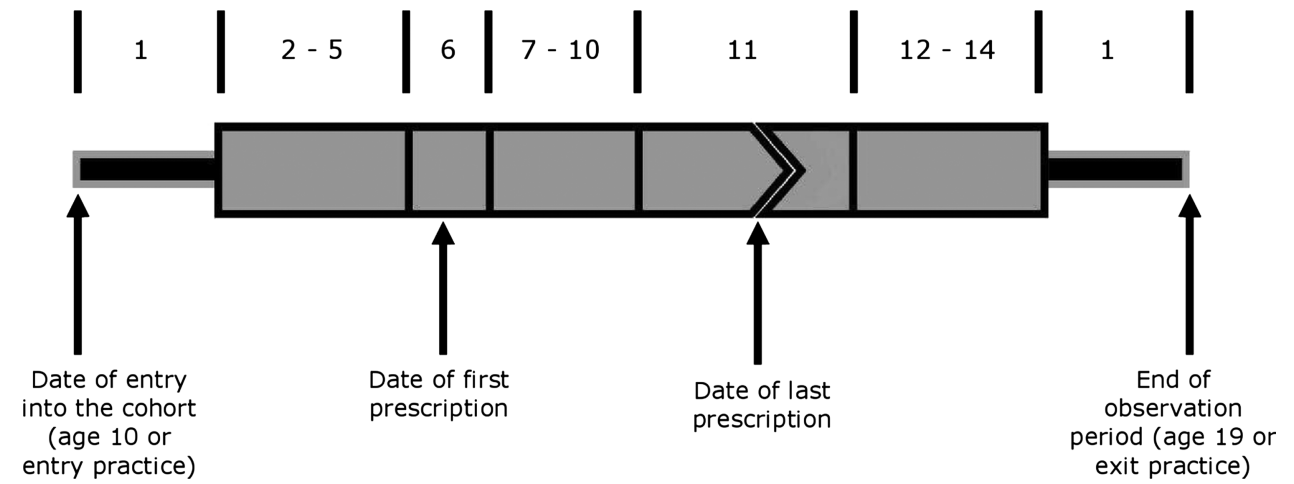

Figure 1 Risk periods included in self-controlled case series analysis for attempted suicide, suicidal ideation and self-harm and suicide. 1=baseline; 2-5=1 month pre-exposure periods; $6=$ prescription day; $7-10=$ four 1 week exposure periods; $11=$ remainder of antidepressant exposure; 12-14=three 1-month washout periods.

\section{RESULTS}

There was a total follow-up time of 4190410 personyears of 10-year-olds to 18-year-olds in THIN. In total, 81 young people were identified with a record of a completed suicide, 1496 young people with a record of attempted suicide, 1178 young people with a record of suicidal ideation, and 2361 with a record of intentional self-harm. Of the young people with completed suicides, $30 \%$ were women, compared to $60 \%, 73 \%$ and $74 \%$ of young people with a record of attempted suicide, suicidal ideation or self-harm, respectively (table 1 ). There was no significant difference in age of first event between the different outcomes. The data were complete for all variables except Townsend scores, which were missing for $92(2 \%)$ persons. Owing to the small numbers, we were not able to analyse antidepressants other than SSRIs or TCAs.

\section{Attempted suicide, suicidal ideation and self-harm}

For attempted suicide, suicidal ideation and self-harm, there were similar patterns between young people who were prescribed SSRIs and TCAs (table 2 and figure 2A-C): there was an upward trend in IRR during pre-exposure; a peak on the day of prescription; a stable or slightly increased rate ratio during the first weeks of prescription; and during the washout period, the levels decreased again. The increase on prescription day was highest for young people with a record of suicidal ideation (SSRIs: IRR $=33.4,95 \%$ CI 23.6 to 47.4 ; TCAs: IRR $=14.0,95 \%$ CI 6.8 to 28.8). There were no significant differences between IRRs for any of the event types for any risk periods. Patterns were similar between individual SSRIs (fluoxetine, citalopram, sertraline and paroxetine, results in the appendix).

The IRR for each type of event has a strong relation with age. When compared to 15-year-olds to 16-year-olds, 17 -year-olds to 18-year-olds are twice as likely to attempt suicide (IRR=1.90, 95\% CI 1.6 to 2.3 and IRR=2.1, 95\% CI 1.7 to 2.5 for SSRIs and TCAs, respectively), but those between 10 and 12 years old are less likely to attempt suicide (IRR=0.3, 95\% CI 0.2 to 0.4 and IRR=0.2, 95\% CI 0.1 to 0.2 for SSRIs and TCAs, respectively). Patterns were similar for the other outcomes.

\begin{tabular}{|c|c|c|c|c|c|}
\hline & $\begin{array}{l}\text { Completed } \\
\text { suicide } 81\end{array}$ & $\begin{array}{l}\text { Attempted } \\
\text { suicide } 1496\end{array}$ & $\begin{array}{l}\text { Suicidal } \\
\text { ideation } 1178\end{array}$ & Self-harm 2361 & $\begin{array}{l}\text { General population } \\
952892\end{array}$ \\
\hline Girls (\%) & $24(29.6)$ & $1089(72.8)$ & $708(60.1)$ & $1752(74.2)$ & $461610(48.4)$ \\
\hline Number of taking ADs (\%) & $19(23.1)$ & $527(36.6)$ & $578(52.8)$ & $128(5.7)$ & 27632 (2.9) \\
\hline Number of depressed (\%) & $21(25.9)$ & $728(48.7)$ & 819 (69.5) & $173(7.3)$ & $41101(4.3)$ \\
\hline \multicolumn{6}{|l|}{$\begin{array}{l}\text { Number of Townsend } \\
\text { score }(\%)\end{array}$} \\
\hline 1 (most affluent) & $20(24.7)$ & $266(17.8)$ & $193(16.4)$ & 442 (18.7) & 227178 (23.8) \\
\hline 2 & $5(6.2)$ & $240(16.0)$ & $202(17.2)$ & 405 (17.2) & $198686(20.9)$ \\
\hline 3 & $13(16.1)$ & $286(19.1)$ & $236(20.0)$ & $452(19.1)$ & $184934(19.4)$ \\
\hline 4 & $25(30.9)$ & 364 (24.3) & $283(24.0)$ & $571(24.2)$ & 169792 (17.8) \\
\hline 5 (most deprived) & $16(19.8)$ & $316(21.1)$ & $241(20.5)$ & $446(18.9)$ & $120116(12.6)$ \\
\hline $\begin{array}{l}\text { Median age in years at } \\
\text { (first) event }(5-95 \%)\end{array}$ & $16.8(12.0-18.8)$ & $16.5(12.9-18.7)$ & $16.7(12.0-18.7)$ & $15.9(12.6-18.7)$ & - \\
\hline $\begin{array}{l}\text { Median time in study in } \\
\text { years }(5-95 \%)\end{array}$ & $3.5(0.2-8.4)$ & $5.5(1.4-9.0)$ & $5.9(1.5-9.0)$ & $5.9(1.5-9.0)$ & - \\
\hline
\end{tabular}




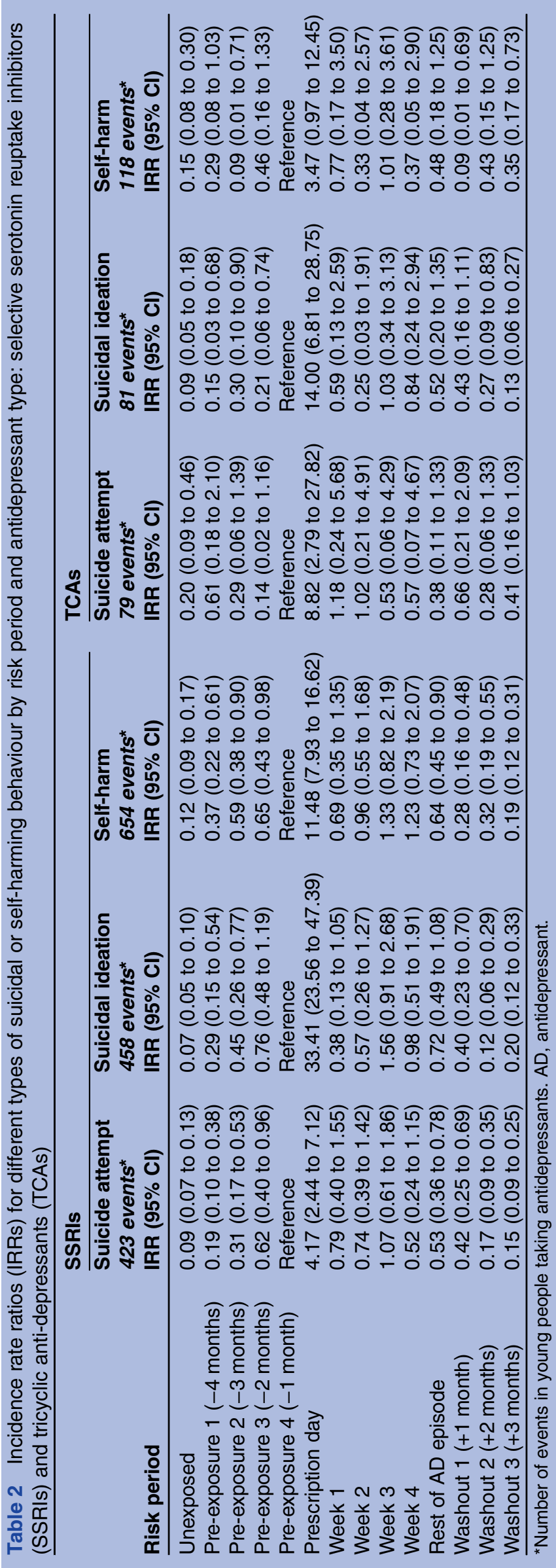

There were no statistically-significant differences between boys and girls for either SSRIs or TCAs (results not shown). A small group of 25 patients had a prescription for an antidepressant and a primary diagnosis other than depression (obsessive compulsive disorder (OCD) or anxiety). Owing to the small size of this group, we did not perform a subgroup analysis. Finally, restricting the analyses to regular consulters (those who consulted at least five times during the first 4 weeks of prescription) did not alter the effect estimates. Of the young people with a record of attempted suicide or intentional selfharm, 33\% were regular consulters, compared to $49 \%$ of young people with a record of suicidal ideation.

\section{Suicide}

Using an expected suicide rate of 3.28 (95\% CI 3.12 to 3.43) per 100000 person-years in the UK population of 10-year-olds to 18-year-olds, ${ }^{29}$ we would expect 137 (95\% CI 131 to 144) completed suicides in our study population. However, $41 \%$ of suicides registered by the Office of National Statistics were of undetermined intent, leaving $59 \%$ or 81 (95\% CI 77 to 85 ) expected suicides that received a verdict by a coroner. This estimate corresponds to the number of 81 completed suicides we identified between 1995 and 2009 within the THIN database. Of the 81 young people with a completed suicide, 21 $(26 \%)$ had a prior record of a depression diagnosis or depression symptoms. Nineteen young people (23\%) were taking antidepressants in the year before their suicide, and $11(14 \%)$ were still taking them at the time of or shortly before their suicide. There was also a high proportion of young people with (a history of) behaviour disorders $16(20 \%)$, a history of self-harm $8(10 \%)$, a psychiatric referral 19 (23\%), a hyperkinetic disorder $5(6 \%)$ or an eating disorder $3(4 \%)$.

There were no completed suicides within the risk periods for antidepressants other than SSRIs (table 3). Eleven (14\%) completed suicides were within the risk periods. Similar to the results from the data on suicide-related events, IRR was highest on the day of prescription (IRR $=42.5,95 \%$ CI 4.5 to 403.4 ). There were no events in the first 2 weeks of the SSRI episode, but there was an increased rate ratio in week 3 (IRR=8.0, 95\% CI 0.8 to 76.7 , based on a single case) and a statistically significant increase in week 4 (IRR $=11.3,95 \%$ CI 1.1 to 115.6, though based on two cases). After the fourth week of the SSRI episode, IRR decreased and returned to baseline levels during washout. There were no significant differences between age groups.

\section{DISCUSSION}

Overall, there are no systematic differences between TCAs and SSRIs in IRRs for attempted suicide, suicidal ideation or intentional self-harm, and apart from an increase common to both TCAs and SSRIs on the day of prescription, rates were not statistically significantly different from pre-exposure levels. The pattern of IRRs for 

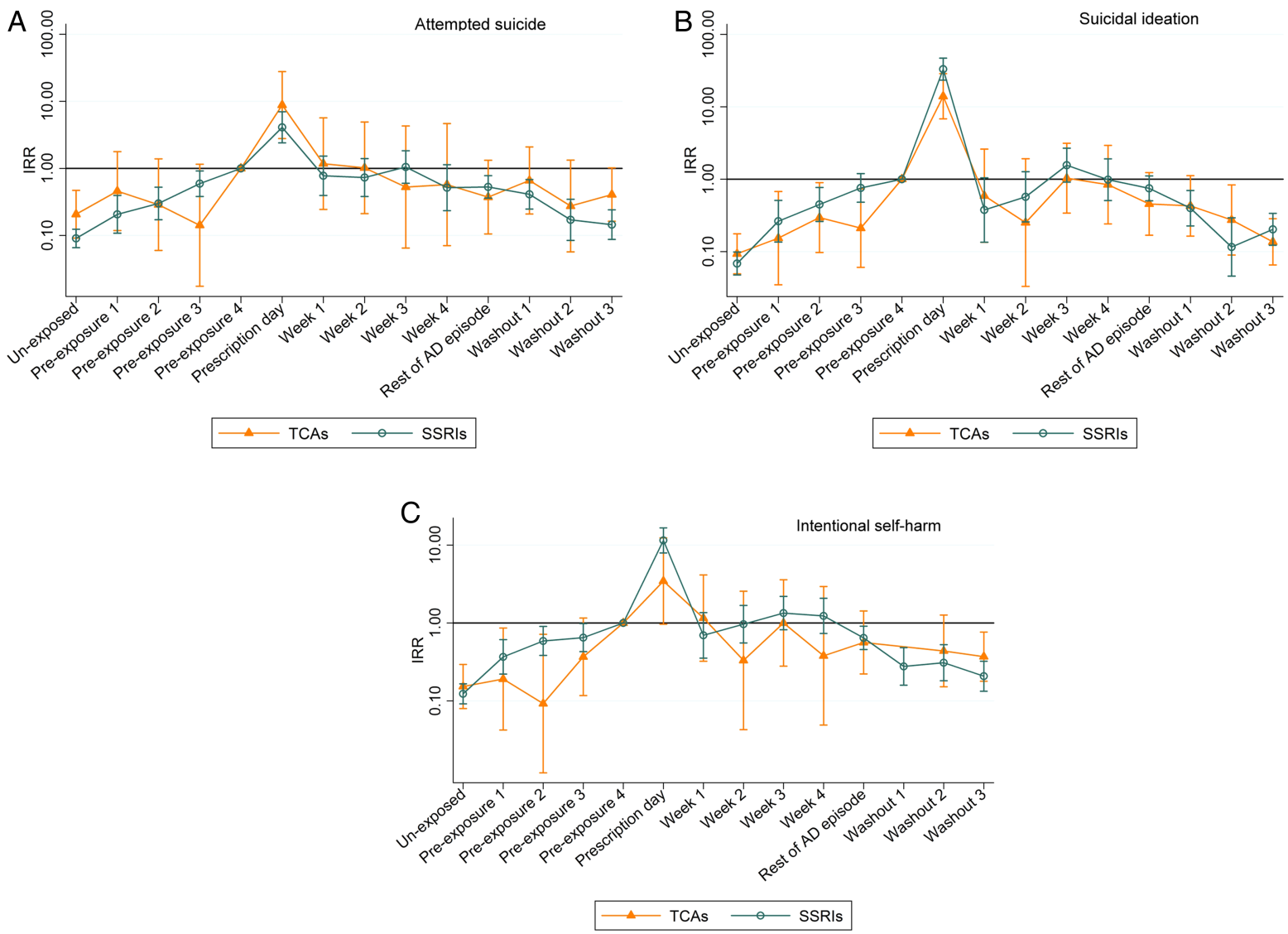

Figure 2 Incidence rate ratios for (A) attempted suicide, (B) suicidal ideation and (C) self-harm for tricyclic antidepressants and selective serotonin inhibitors. The month before a prescription was issued (pre-exposure 4) was used as the reference.

completed suicides for the 11 people prescribed SSRIs was similar to that found in attempted suicide, suicidal ideation and self-harm. However, concerns regarding

Table 3 Incidence rate ratios (IRRs) for completed suicide by risk period and age

\begin{tabular}{|c|c|c|}
\hline Risk period & $\begin{array}{l}\text { Censoring model } \\
\text { IRR }(95 \% \mathrm{Cl})\end{array}$ & $\begin{array}{l}\text { Number of } \\
\text { deaths* }\end{array}$ \\
\hline Prescription day & 42.52 (4.48 to 403.43$)$ & 5 \\
\hline Week 1 & No events & 0 \\
\hline \multicolumn{3}{|l|}{ Week 2} \\
\hline Week 3 & 8.00 (0.84 to 76.71$)$ & 1 \\
\hline Week 4 & 11.25 (1.09 to 115.58$)$ & 2 \\
\hline $\begin{array}{l}\text { Rest of } A D \\
\text { episode }\end{array}$ & $5.42(0.57$ to 51.94$)$ & 1 \\
\hline Washout 1 & $2.27(0.24$ to 21.76$)$ & 1 \\
\hline Washout2 & 2.08 (0.22 to 19.69$)$ & 1 \\
\hline Age groups & & $\begin{array}{l}\text { Number of total } \\
\text { deaths } \dagger\end{array}$ \\
\hline 10 to 12 & 0.61 (0.21 to 1.77$)$ & 8 \\
\hline 13 to 14 & $1.14(0.45$ to 2.90$)$ & 15 \\
\hline 15 to 16 & Reference & 21 \\
\hline 17 to 18 & $0.41(0.12$ to 1.39$)$ & 37 \\
\hline \multicolumn{3}{|c|}{$\begin{array}{l}\text { *Number of suicides during risk periods (only includes youths who } \\
\text { were taking antidepressants at the time of suicide, or those who } \\
\text { had recently stopped). } \\
\text { †Number of suicides by age category. }\end{array}$} \\
\hline
\end{tabular}

antidepressants need to be weighed against the risk of untreated depression.

Pre-exposure IRRs for attempted suicide, suicidal ideation and self-harm appeared to be marginally higher, though not statistically significant, for SSRIs compared to TCAs. This could be because young people who are deemed to be more at risk of suicide-related events could preferentially be prescribed SSRIs over TCAs, given TCAs' toxicity in overdose. ${ }^{17}$

The high IRRs on the day of antidepressant prescription for all three non-fatal outcomes could be an artefact of GP-recording behaviour. Rather than the antidepressant causing the suicide-related events, the event is an indication for the GP to prescribe the drug and to record depression in the electronic records on the same day. As antidepressants should only be prescribed by child and adolescent psychiatrics (2005 NICE guidelines $^{6}$ ), this artefact could also arise when GPs continue a prescription started in secondary care and record the initial indication when first prescribing this drug.

Up to the fourth week of prescription, the IRRs for attempted suicide, suicidal ideation and self-harm remained around the levels experienced during the preexposure period. Considering that suicide-related events are common in young depressed people, some suicidal and self-harm events would be expected irrespective of whether SSRIs are prescribed or not. ${ }^{30}$ The rate of 
suicide-related events decreased when the prescriptions were stopped. Given the nature of the data, it is difficult to know whether the SSRIs were causing suicide-related events and these events improved when the SSRIs were discontinued, or if the SSRIs were discontinued when the young person's depression (and as a consequence the risk of suicide-related events) improved.

There are three possible explanations for the slightly increased IRRs during the first month of prescription. One is that the SSRIs fail to relieve the risk of suicide-related events associated with depression because of a lag in the antidepressant effect, an incomplete response, or a treatment-resistant depression. It is known that SSRIs take a couple of weeks to reach their full antidepressant effects and hence reduce the risk of suicide-related events. $^{27}$

A second possibility is that the SSRIs generate a novel set of suicidal emotions or behaviours. Early improvements in clinical depression can lead to a person acting on existing suicidal feelings. This activation syndrome has been described for TCAs and SSRIs, and is widely recognised by psychiatrists as well as the FDA. ${ }^{31}{ }^{32}$ While patients might be demotivated and demoralised at the height of their depression, when they start treatment they become more active during the first weeks of taking the drug. During this time, the antidepressant effect of the medication will not have reached its full effect, resulting in persistent depression but simultaneous increased activity. This could lead to a greater risk of suicide-related events until the full effects of antidepressants are realised a few weeks later. ${ }^{31}$

Several studies and systematic reviews have shown an age effect in the risk of suicide-related events with the use of SSRIs. In adults and the elderly, the risk is neutral or SSRIs show a protective effect, while in adolescents and young adults there appears to be an increased risk of suicide-related events. 91533

Although we did not find a statistically significantly sustained increase in suicide-related events with either SSRIs or TCAs, negative outcomes did not appear to be decreased either-although our study was not designed to assess this. This is in line with the Cochrane reviews on both drug groups: the review on TCAs concludes that these drugs are not useful in treating depression in prepubertal children, and that there is only marginal evidence to support the use of TCAs in adolescents, although the magnitude of the effect is likely to be moderate at best. ${ }^{3}$ Similarly, it is unclear what the effect is of SSRIs on suicide completion. Although evidence from clinical trials implies an increased risk of suicide-related outcomes (but not completed suicide), the evidence for this association is of low quality. ${ }^{34}$

\section{Comparison to other studies}

Our results build on the findings of Schneeweis et $a l^{13}$ They found no statistically significant differences in the relative risk of attempted and completed suicide between different types of antidepressants (fluoxetine, citalopram, fluvoxamine, paroxetine, sertraline and TCAs) when examining 266 attempted and three completed suicides. Moreover, an ecological study found no change in rates of completed suicides or hospital admissions for self-harm following the CSM advice, ${ }^{35}$ suggesting that there is no, or only a weak, relationship with antidepressant prescriptions. Our findings are also similar to those of Simon $e t a l^{36}$ who used computerised health plan records and reported the highest rates for attempted suicide in the month before prescription, rather than after start of the prescription. Finally, a meta-analysis ${ }^{37}$ found that, of 27 paediatric randomised controlled trials on antidepressants prescribed for MDD, OCD and non-OCD anxiety disorders, the risk of suicidal ideation or attempt among patients on placebo was greater in trials assessing MDD. Although this difference in baseline risk for suicide-related events was not statistically-significant, it could indicate that a part of the association between antidepressants and suicide-related events can be explained by the underlying disease, rather than the drug. Importantly, the authors concluded that relative to placebo, the benefits (though only modest in MDD) outweigh the risks from suicidal ideation/suicide attempts. Owing to the small numbers of patients with primary diagnoses of OCD and anxiety disorders, we could not repeat the meta-analysis's subgroup comparison.

\section{Main strength and limitations}

The main strength of this study is its sample size that enables the examination of outcomes separately by completed and attempted suicide, suicidal ideation and selfharm, as well as by individual antidepressants. However, even in using a database as large as THIN, we could identify only a small number of completed suicides, leading to limited power in that analysis. Similarly, power was limited for analysing individual antidepressants, which are presented in the appendix. Nevertheless, the use of the SCCS method allows us to control for timeindependent confounders, making our estimations more robust.

A limitation to our study is that the THIN database only provides data on antidepressant prescriptions. We do not know whether the prescriptions were dispensed, or whether the patients adhered to the prescription. However, though it is known that adherence levels are around $50 \%$ for young people taking SSRIs, and even lower for TCAs, ${ }^{38}$ our data do represent a real-life situation. Moreover, by assessing episodes of antidepressant prescription, we take account of multiple prescriptions per patient, which increases the likelihood of adherence, as we expect patients who are not taking their medication will not return for a new prescription.

Moreover, it is known that suicide-related events are often missed in clinical assessment. ${ }^{39}$ However, it is quite likely that the most severe forms (attempted suicides and severe suicidal ideation) are most likely to be recorded by clinicians. Also, in using a self-controlled 
design, we decrease the chance of misclassifying controls. There is some suggestion that antidepressants might specifically increase suicide-related events in patients who did not experience these events prior to starting antidepressant treatment. Owing to the variation in the clinicians' assessment and recording of (the absence of) suicide-related events, we could not examine this hypothesis using this database. Furthermore, the relatively low number of young people who had a prescription for an antidepressant at the time of their suicide-related event limits the interpretation of our results. However, Windfuhr et $a l^{29}$ also found that mental health service contact was low in juveniles who committed suicide: only $14 \%$ contacted services in the year before they died. Finally, we were not able to account for changes in depression severity over time as this is poorly recorded.

\section{CONCLUSION}

Our study shows that there are similar IRR patterns for attempted suicide, suicidal ideation and self-harm for SSRIs and TCAs. Also, the pattern for completed suicides associated with SSRI prescriptions is similar, though there are no records of completed suicides within our predefined risk periods for TCAs. Although the CSM's warning was a sensible cautionary recommendation at the time, it appears that the current line of evidence suggests a reverse causality: it is the underlying depression that leads to suicide-related events and the prescription of antidepressants, although a causal effect of SSRIs or no effect at all cannot be ruled out. Moreover, even if antidepressant drugs would temporarily increase the risk of suicide-related events in young persons, the risk posed by untreated depression is far greater. In conclusion, our results indicate that the association of suicide-related events associated with antidepressants occurs primarily around the day of prescription, suggesting depression severity and GP-recording behaviour as the culprit rather than antidepressants, and thus warrants a re-evaluation of the current guidelines regarding the prescription of SSRIs in primary care.

\section{RECOMMENDATION}

Our results are not definitive, and due to the rare nature of the outcome and the intricacies of the problem studied, it is difficult to think of a single study or study design that will be able to provide a satisfying answer to the problem at hand. It is possible that the creation of a pragmatic registry, similar to that proposed by van Staa et $a l^{40}$ in their pragmatic randomised trial, will allow for active pharmacovigilance. Such a system would, at low cost and with no additional burden on clinician, health service or patient time, facilitate long-term, anonymous, unobtrusive follow-up for major clinical outcomes. As such, clinicians would be prompted to monitor and record (the absence of) suicide-related events and ideation more regularly and closely, using similar outcome measurements as those used in clinical trials, as well as (changes in) depression severity.

Acknowledgements The authors would like to thank the general practitioners who contributed data to THIN.

Contributors All authors were responsible for study conception and design. LPW was responsible for data analysis. LPW drafted the manuscript. All authors participated in the interpretation of data, critical revision and final approval of the manuscript.

Funding The work was supported by a PhD studentship from the National Institute of Health Research English National School for Primary Care Research. The funding organisation had no role in the design and conduct of the study; collection, management, analysis and interpretation of data; and preparation, review or approval of the manuscript.

Competing interests LPMMW had financial support in the form of a studentship from the National Institute of Health Research School for Primary Care Research for the submitted work.

Ethics approval National Health Service South-East Multicenter Research Ethics Committee.

Provenance and peer review Not commissioned; externally peer reviewed.

Data sharing statement Examples of statistical code used for the self-controlled case series method (including the adapted method for dealing with censored data) are available from the Open University website. Statistical code is available from the corresponding author.

Open Access This is an Open Access article distributed in accordance with the Creative Commons Attribution Non Commercial (CC BY-NC 3.0) license, which permits others to distribute, remix, adapt, build upon this work noncommercially, and license their derivative works on different terms, provided the original work is properly cited and the use is non-commercial. See: http:// creativecommons.org/licenses/by-nc/3.0/

\section{REFERENCES}

1. Kessler RC, Avenevoli S, Ries, et al. Mood disorders in children and adolescents: an epidemiologic perspective. Bioll Psychiatry 2001;49:1002-14

2. Heron M. Deaths: leading causes for 2007. Natl Vital Stat Rep 2011:59:1-95

3. Hazell P, O'Connell D, Heathcote D, et al. Tricyclic drug for depression in children and adolescents. Cochrane Database Syst Rev 2010;(4):CD002317.

4. Wijlaars LP, Nazareth I, Petersen I. Trends in depression and antidepressant prescribing in children and adolescents: a cohort study in the health improvement network (THIN). PLOS ONE 2012;7: e33181.

5. Gunnell D, Saperia J, Ashby D. Selective serotonin reuptake inhibitors (SSRIs) and suicide in adults: meta-analysis of drug company data from placebo controlled, randomised controlled trials submitted to the MHRA's safety review. BMJ 2005;330:385.

6. National Institute for Health and Clinical Excellence. CG28 depression in children and young people: NICE guideline. London: National Institute for Health and Clinical Excellence, 2005.

7. FDA. Suicidality in children and adolescents being treated with antidepressant medications. US Food and Drug Administration. 2004. http://www.fda.gov/Drugs/DrugSafety/ PostmarketDrugSafetylnformationforPatientsandProviders/ DrugSafetylnformationforHeathcareProfessionals/ PublicHealthAdvisories/ucm161679.htm (accessed 29 June 2011).

8. Whittington CJ, Kendall T, Fonagy $P$, et al. Selective serotonin reuptake inhibitors in childhood depression: systematic review of published versus unpublished data. Lancet 2004;363:1341-5

9. Hammad TA, Laughren T, Racoosin J. Suicidality in pediatric patients treated with antidepressant drugs. Arch Gen Psychiatry 2006;63:332-9.

10. Rothman KJ, Michels KB. The continuing unethical use of placebo controls. N Engl J Med 1994;331:394-8.

11. Grosso A, Douglas I, MacAllister R, et al. Use of the self-controlled case series method in drug safety assessment. Expert Opin Drug Saf 2011;10:337-40. 
12. Gibbons RD, Brown $\mathrm{CH}$, Hur $\mathrm{K}$, et al. Early evidence on the effects of regulators' suicidality warnings on SSRI prescriptions and suicide in children and adolescents. Am J Psychiatry 2007;164:1356-63.

13. Schneeweiss S, Patrick AR, Solomon DH, et al. Comparative safety of antidepressant agents for children and adolescents regarding suicidal acts. Pediatrics 2010;125:876-88.

14. Jick $\mathrm{H}$, Kaye JA, Jick SS. Antidepressants and the risk of suicidal behaviors. JAMA 2004;292:338-43.

15. Olfson M, Marcus SC, Shaffer D. Anti-depressant drug therapy and suicide in severely depressed children and adults-a case-control study. Arch Gen Psychiatry 2006;63:865-72.

16. Gibbons RD, Brown $\mathrm{CH}$, Hur K, et al. Suicidal thoughts and behavior with antidepressant treatment: reanalysis of the randomised placebo-controlled studies of fluoxetine and venlafaxine. Arch Gen Psychiatry 2012;69:580-7.

17. Didham RC, McConnell DW, Blair HJ, et al. Suicide and self-harm following prescription of SSRIs and other antidepressants: confounding by indication. Br J Clin Pharmacol 2005;60:519-25.

18. Bourke A, Dattani H, Robinson M. Feasibility study and methodology to create a quality-evaluated database of primary care data. Inform Prim Care 2004;12:171-7.

19. Booth N. What are the read codes? Health Libr Rev 1994;11:177-82.

20. Khan NF, Harrison SE, Rose PW. Validity of diagnostic coding within the general practice research database: a systematic review. $\mathrm{Br} J$ Gen Pract 2010;60:e128-36.

21. Arana A, Wentworth CE, yuso-Mateos JL, et al. Suicide-related events in patients treated with antiepileptic drugs. $N$ Engl J Med 2010;363:542-51.

22. Davé S, Petersen I. Creating medical and drug code lists to identify cases in primary care databases. Pharmacoepidemiol Drug Saf 2009;18:704-7.

23. British National Formulary. 60th edn. London: BMJ Group, RPS Publishing, 2010.

24. The NHS Information Centre PaPCS. Prescription compliance: a review of the proportion of prescriptions dispensed. 7-9-2011.

25. Whitaker HJ, Farrington CP, Spiessens B, et al. Tutorial in biostatistics: the self-controlled case series method. Stat Med 2006;25:1768-97.

26. Whitaker $\mathrm{HJ}$, Hocine MN, Farrington CP. The methodology of self-controlled case series studies. Stat Methods Med Res 2009;18:7-26.
27. Taylor MJ, Freemantle N, Geddes JR, et al. Early onset of selective serotonin reuptake inhibitor antidepressant action: systematic review and meta-analysis. Arch Gen Psychiatry 2006;63:1217-23.

28. Farrington $\mathrm{CP}$, Whitaker $\mathrm{HJ}$, Hocine MN. Case series analysis for censored, perturbed, or curtailed post-event exposures. Biostatistics 2009;10:3-16.

29. Windfuhr K, While D, Hunt I, et al. Suicide in juveniles and adolescents in the United Kingdom. J Child Psychol Psychiatry 2008;49:1155-65.

30. Bridge JA, Goldstein TR, Brent DA. Adolescent suicide and suicidal behavior. J Child Psychol Psychiatry 2006;47:372-94.

31. Sinclair LI, Christmas DM, Hood SD, et al. Antidepressant-induced jitteriness/anxiety syndrome: systematic review. Br J Psychiatry 2009;194:483-90.

32. Culpepper L, Davidson JR, Dietrich AJ, et al. Suicidality as a possible side effect of antidepressant treatment. J Clin Psychiatry 2004;65:742-9.

33. Barbui C, Esposito E, Cipriani A. Selective serotonin reuptake inhibitors and risk of suicide: a systematic review of observational studies. CMAJ 2009;180:291-7.

34. Hetrick SE, McKenzie JE, Cox GR, et al. Newer generation antidepressants for depressive disorders in children and adolescents. Cochrane Database Syst Rev 2012;11:CD004851.

35. Wheeler BW, Gunnell D, Metcalfe C, et al. The population impact on incidence of suicide and non-fatal self harm of regulatory action against the use of selective serotonin reuptake inhibitors in under 18s in the United Kingdom: ecological study. BMJ 2008;336:542-5.

36. Simon GE, Savarino J, Operskalski B, et al. Suicide risk during antidepressant treatment. Am J Psychiatry 2006;163:41-7.

37. Bridge JA, lyengar $\mathrm{S}$, Salary $\mathrm{CB}$, et al. Clinical response and risk for reported suicidal ideation and suicide attempts in pediatric antidepressant treatment: a meta-analysis of randomized controlled trials. JAMA 2007;297:1683-96.

38. Barbui C, Hotopf M, Freemantle N, et al. Selective serotonin reuptake inhibitors versus tricyclic and heterocyclic antidepressants: comparison of drug adherence. Cochrane Database Syst Rev 2000; (4):CD002791.

39. Fitzpatrick C, Abayomi NN, Kehoe A, et al. Do we miss depressive disorders and suicidal behaviours in clinical practice? Clin Child Psychol Psychiatry 2012;17:449-58.

40. van Staa T, Goldacre B, Gulliford M, et al. Pragmatic randomised trials using routine electronic health records: putting them to the test. BMJ 2012;344:e55 